\title{
El Apéndice de la Colección de obras impresas y manuscritas [1853] de Pedro de Angelis. Una reconstrucción de la parte etnolingüística.
}

The Appendix to Pedro de Angelis' Colección de obras impresas y manuscritas [1853] A special collection in ethnolinguistics reconstructed.

Franz Obermeier*

Resumen: En este artículo se presenta un documento sobre los inicios de la etnolingüística argentina en el siglo XIX, el Apéndice a la colección de Pedro de Angelis que contenía documentos etnolingüísticos importantes conformado por manuscritos e impresos, principalmente escritos en las reducciones jesuíticas del Paraguay. La creación de redes de contacto entre investigadores argentinos interesados en el tema comenzó en el siglo XIX. Esto se inició con el interés de coleccionistas que querían poseer "curiosidades", como primeros manuscritos de lenguas indígenas u obras gramaticales y vocabularios impresos por los jesuitas. La figura más prominente entre éstos es el coleccionista de origen italiano Pedro de Angelis, que desarrolló su interés lingüístico hasta un alto nivel. De Angelis vendió la mayor parte de su colección al Emperador del Brasil (ahora en la Biblioteca Nacional en Río de Janeiro) pero conservó una pequeña colección etnolinguiística de gran calidad que vendió separadamente a algunos amigos en Buenos Aires. Esa parte es solo conocida a través de un Apéndice, publicado en Buenos Aires, para completar su principal bibliografía hecha para la venta en Brasil en 1853. La propia contribución de De Angelis a la lingüística ha sido completamente descuidada hasta ahora.

Palabras clave: Pedro de Angelis, etnolingüística del siglo XIX, manuscritos, impresos

\footnotetext{
* Universitätsbibliothek Kiel, Alemania. E-mail: obermeier@ub.uni-kiel.de
} 


\begin{abstract}
This article presents an important document about the beginnings of Argentine ethnolinguistics in the 19th century, the Apendice to the Angelis collection, which contained important etnolinguistic documents mainly manuscripts written by Jesuits in colonial times in the so called reducciones. Networking among interested Argentinean researchers also began in the mid-19th century. Initially it consisted mainly in the interest of collectors who wanted to possess special curiosities, early manuscripts in indigenous languages or the rare printed grammatical works and vocabularies of the Jesuits. The most prominent figure amongst collectors is the Italo-argentine collector Pedro de Angelis with a whose linguistic interest at high level. He sold his collection (the main part went to Brazil, now in the Biblioteca Nacional in Rio). Angelis deliberately retained his ethnolinguistic collection, which he later sold to a few friends only in Buenos Aires (known only through an "Apendice", published in Buenos Aires to complete his main bibliography made for the sale in Brazil). Angelis' own contribution in linguistics has been completely neglected until now.
\end{abstract}

Keywords: Pedro de Angelis, ethnolinguistics 19th century, manuscripts, imprints

Recibido: 5 de marzo de 2017.

Evaluado: 21 de abril de 2017. 


\section{Introducción}

La biografía de Pedro de Angelis (Nápoles, 1784 - Buenos Aires, 1859) es bien conocida para los que se interesan por la historia de la ciencia y del periodismo en Argentina en el siglo XIX. Podemos aquí prescindir de repetir los datos conocidos en detalle. Llegado al Río de la Plata vivió en Montevideo a partir de 1826, por sugerencia del presidente de las Provincias Unidas del Río de la Plata Bernardino Rivadavia. Después lo hizo en Buenos Aires, donde intentó establecerse como publicista de varias revistas, impresor y archivero. Fue durante 12 años, entre 1840 y 1852, Segundo Archivero del Archivo General de la Provincia de Buenos Aires, en realidad su Director, porque el mismo era ya muy mayor (Sabor, 1995, p. 88), hecho que sus oponentes, por supuesto, han usado con mala intención para insinuar que parte de su colección privada de manuscritos provendría de dominio público. Los pormenores de la constitución de esa colección no son bien conocidos, pero en ese siglo había en la zona rioplatense algunas de importancia histórica, en manos privadas. La de Saturnino Segurola (1776-1854) por ejemplo, contenía también documentos históricos originales. 'Había en ese tiempo coleccionistas que habían conseguido manuscritos que hoy serían patrimonio histórico nacional a través de compra o intercambio. No se encontraban prácticamente archivos públicos y es importante observar, que en algunos casos, esos coleccionistas han ciertamente conservado documentos únicos que estaban predestinados a la destrucción, como lo hizo Francisco Javier Brabo (1825-1913) con los documentos jesuíticos sudamericanos en España (Brabo, 1872a y 1872b).

Viene recordado De Angelis principalmente por una obra cuyo trabajo pionero fue reconocido más tarde, la primera edición de fuentes de la región rioplatense (De Angelis, 1836-1837). Es muy probable que la publicación fuera inspirada por Juan María Gutiérrez, más no está documentada. Además, De Angelis publicó un gran número de folletos actualizados dia-riamente, muchos estudios de carácter historiográfico, como vemos en la bio-bibliografia de Sabor (1995). Es menos conocido su interés por la etnolingüística que vamos a tratar en este artículo analizando y editando un documento precisamente sobre la parte etnolingüística de su colección.

Después de años de negociación y ya en Montevideo (anticipando un decreto que lo exilió de Buenos Aires por el apoyo marcado a Rosas en sus revistas) vendió su única colección de libros, mapas y manuscritos al Emperador del Brasil Pedro II en 1853. Hoy se encuentra en la Biblioteca Nacional del Brasil. Solo parte de esos documentos fueron publicados por Jaime Cortesão entre 1951 y 1969. Hay un catálogo de los mapas (Leal Feitosa Coelho, 2001, pp. 223-256). Un proyecto de digitalización de esas fuentes, junto con la Argentina que está poco avanzado. ${ }^{2}$

En un trabajo reciente hemos hecho una evaluación de una parte olvidada de su colección, la que no vendió al Emperador del Brasil y que podemos llamar la parte etnolingüística de la misma, dispersa con una data desconocida (probablemente antes de 1856) a través de una venta a individuos en Buenos Aires (Obermeier, 2015, pp. 69-90). Aquí presentamos por primera vez, en edición comentada en español, un catálogo de esa

\footnotetext{
${ }^{1}$ Segurola fue director de la La Biblioteca Pública de Buenos Aires, fundada en 1821, antecesora directa de la Biblioteca Nacional. El fondo manuscrito de la colección de Segurola antes en la Biblioteca Nacional está hoy por decreto, en el Departamento Documentos Escritos del Archivo General de la Nación en Buenos Aires.

http://bndigital.bn.br/projetos/angelis/spa/proyecto.html.
}

5 Franz Obermeier. El Apéndice de la Colección de obras impresas y manuscritas... 3-27. 
colección que resalta su valor único. Se trata de un Apéndice al catálogo de venta de dicha colección del que solo existen pocos ejemplares, por ello casi desconocido, excepto en menciones bibliográficas.

El catálogo de venta de la colección que llegó después al Brasil fue una de las primeras bibliografías importantes de la historia rioplatense. Se publicó en 1853 y ciertamente se hallan pocos ejemplares. ${ }^{3}$ El Apéndice se refiere a esa obra, pero De Angelis lo publicó con una tirada limitada, y lo distribuyó a algunos amigos personales y posibles compradores en Buenos Aires. Hoy es extremadamente raro.

Sabor (1995, p. 246) menciona tres ejemplares de ese Apéndice, todos encuadernados con el catálogo de venta. "Algunos de los ejemplares del catálogo [de venta citado encima] que se conservan en las bibliotecas argentinas no poseen el Apéndice, que en ciertos casos (por ejemplo, Biblioteca del Congreso de la Nación) ha sido agregado manuscrito. Los ejemplares del Museo Mitre y de la Biblioteca de la Universidad Nacional de La Plata, Colección Farini, lo tienen”. El ejemplar del Museo Mitre fue robado, la Biblioteca del Congreso no ha respondido a mi pregunta si todavía lo tienen y por eso hemos usado el ejemplar de la Biblioteca de la Universidad Nacional de La Plata que nos ha facilitado el acceso. Agradecemos el gesto.

Vamos antes a dar una transcripción completa de las tres páginas. El Apéndice contiene libros impresos y manuscritos. Los libros son fáciles de identificar en cuanto al título, más como De Angelis no usó ex-libris o inscribió su nombre en sus ejemplares, sus libros todavía existentes no pueden ser identificados, a no ser que indirectamente. En esa parte de los libros impresos del Apéndice solo hay un error de data que es un error evidente de impresión. Más importante es la parte de los manuscritos únicos que De Angelis ha poseído. Por eso tentamos una identificación de esos manuscritos mencionados en el Apéndice, presentando aquí nuestros resultados de investigaciones extensas. $^{5}$

Los principales compradores de esa colección fueron Bartolomé Mitre, dedicado a la historiografía argentina después de su carrera política y Manuel Trelles, funcionario público, historiador y coleccionista. La colección de Mitre se conservó en su casa que después de su muerte se transformó en museo (Mitre y Torres, 1909) ${ }^{6}$. La colección de los Trelles fue comprada en 1916 por Enrique Peña, historiador y coleccionista. No tenemos documentos sobre qué títulos compraran Mitre o Trelles, más podemos deducir mucho de los contextos y en el caso de Mitre, de los ejemplares que todavía se conservan en el Museo Mitre.

${ }^{3}$ Hay pocos ejemplares que se conservan hoy. El que sirvió a la negociación con el Brasil está en la Biblioteca del Itamaraty, el Ministério das Relações Exteriores con biblioteca en Rio. Es lógico que no contiene el apéndice, mas notas manuscritas del Conde de Rio Branco. El texto de esa bibliografia está disponible en línea sin el Apéndice: http://www.cervantesvirtual.com/ o Recuperado de http://www.biblioteca.org.ar/libros/300749.pdf.

${ }^{4}$ El Tercer catecismo y exposicion de la doctrina cristiana apareció después del Concilio de Lima en 1585 y no en 1685 como está mencionado erradamente en el Apéndice.

${ }^{5}$ Agradecemos desde ya, a las bibliotecas y bibliotecarios que han ayudado a identificar marcas de proveniencia o datas de adquisición en sus ejemplares.

6 Ver el extracto etnolingüístico del catálogo sin título en http://www.museomitre.gov.ar/pdfs/Catalogo_lenguas_americanas.pdf. Citamos los números de ese último catálogo y si las tenemos las signaturas usadas hoy en el Museo Mitre.

6 Franz Obermeier. El Apéndice de la Colección de obras impresas y manuscritas... 3-27. 
Hemos identificado la mayor parte de esos manuscritos, de algunos sabemos también su paradero actual, sólo permanecen pequeñas dudas sobre un manuscrito que figura en el Apéndice y es listado con título no muy especifico.

\section{Apéndice al catálogo de la biblioteca de D. Pedro de Angelis.}

(Ejemplar de la Universidad de La Plata Colección Juan Ángel Farini).

\section{[Original pág. I]}

\section{OBRAS EN LENGUA GUARANÍ.}

-Tesoro de la lengua guaraní, por el P. Antonio Ruiz de Montoya. Madrid, 1639 en $8^{\circ}$.

-Arte y vocabulario de la lengua guaraní, por el mismo. Madrid, 1640 en $8^{\circ}$.

-Catecismo en lengua Guaraní, por el mismo. Madrid, 1640 en 12.

-Demostración clara, y evidente respuesta á las calumnias nuevamente inventadas contra los religiosos de la Compañía de Jesús de las Provincias del Paraguay, sobre el Catecismo, Oraciones y Doctrina Cristiana de la lengua guaraní, por el P. Díaz Taño. Autógrafo.

-Arte de la lengua guaraní, por el P. Blas Pretovio. (Anagrama de Pablo Restivo) Autógrafo, escrito en 1696.

-Diario del desalojo de los Portugueses de la Colonia, en 1704. Manuscrito.

-De la diferencia entre lo temporal y eterno crisol de

[Original pág. II] desengaños, por el P. Nieremberg, traducido al idioma guaraní por el P. José Serrano. En las Doctrinas del Paraguay, 1705 en fol., con 43 láminas.

-Manuale ad usum Patrum Societatis Jesu Paraquarias, En español y guaraní. Loreto, 1721 en $8^{\circ}$.

-Vocabulario de la lengua guaraní, por el P. Antonio Ruiz de Montoya, aumentado por el P. Pablo Restivo. Santa Maria la Mayor, 1722 en $4^{\circ}$.

-Arte de la lengua guaraní del P. Montoya, reimpreso por el P. Restivo. Santa María la Mayor, 1724 en $4^{\circ}$.

-Explicacion del catecismo en lengua guaraní, por Nicolás Yapuguay. Santa María la Mayor, 1724 en $4^{\circ}$.

-Sermones y ejemplos en lengua guaraní, por el mismo. San Francisco Xavier, 1727 en $4^{\circ}$.

-Ara poru, por el P. Insaurralde. Madrid, 1759, 2 vol. en 12.

-Catecismos varios en lengua guaraní. Manuscrito en $4^{\circ}$.

-Frases selectas de la lengua guaraní. Manuscrito en $4^{\circ}$.

-Compendio de la lengua guaraní. Manuscrito en 12. 
-Diálogos en lengua guaraní. Manuscrito en 12.

-Catecismo en castellano y guaraní, por el P. Bernal. Buenos Aires. 1800 en 12.

-Notas al Catecismo en español y guaraní, publicado por Fray José Bernal, P. Provincial de San Francisco de Buenos Aires. Manuscrito.

-Oficios en lengua guaraní, con las respectivas traducciones en español. Manuscrito en fol.

-Arte breve de la lengua guaraní. Manuscrito en 12.

-Confesionario de la lengua guaraní. Manuscrito en 12.

-Texto de la doctrina cristiana, por el P. Bolaños. Manuscrito en 12.

-Explicación de la doctrina cristiana. Manuscrito en 12.

[Original pág. III]

OBRAS EN LENGUA AIMARÁ.

-Vocabulario de la lengua aimará, por el P. Bertonio, Juli, 1612 en $4^{\circ}$.

-Arte de la lengua aimará, por el mismo. Juli, 1612.

-Arte de la lengua aimará, por el P. Torres Rubio. Lima, 1616 en 12.

-Catecismo de la lengua aimará. Sevilla, 1604 en 12.

-Tercer catecismo y exposición de la doctrina cristiana, Lima, 1685 en $4^{\circ}$. [evidente error para 1585].

-Evangelio de San Lucas en lengua aimará, por Pazos. Londres, 1829 en 12.

\section{OBRAS EN LENGUA QUICHUA.}

-Gramática de la lengua quichuá, ó del Perú, por Fray Domingo de Santo Tomas. Valladolid, 1560 en 12.

-Lexicón de la lengua quichua, por el mismo. Valladolid, 1560 en 12.

-Ritual para los Curas del Perú, por Bocanegra. Lima, 1631 en $4^{\circ}$.

-Arte y vocabulario do la lengua quichua, por el P. Torres Rubio. Lima, 1754 en 12.

\section{OBRAS EN LAS LENGUAS QUICHUA, Y AIMARÁ.}

-Doctrina cristiana. Ciudad do los Reyes. 1584 en $4^{\circ}$.

-Confesionario para los Curas de lndios. Ciudad de los Reyes, 1585 en $4^{\circ}$.

\section{OBRAS EN LENGUA CHILENA.}

-Arte y vocabulario de la lengua de Chile, por el P. Febres. Lima, 1765 en 12.

-Doctrina cristiana. Manuscrito en 12. 
Los impresos son fáciles de identificar y conocidos por todos los especialistas de la primera imprenta argentina en las reducciones o en los libros jesuíticos en general. Hoy en día la mayor parte de las gramáticas impresas y libros de doctrina religiosa se encuentran digitalizados en el proyecto John Carter Brown Library - Indigenous Collection en Providence y disponibles online.

Solo resaltamos el primer libro impreso ${ }^{8}$ en Argentina, el del P. Nieremberg De la diferencia entre lo temporal y eterno, impreso en las reducciones en 1705. Es el ejemplar que hoy a través de la donación de Elisa Peña, hija de Enrique Peña está en el Museo Udaondo en Luján. ${ }^{9}$ Fue comprado por Trelles, después pasó a la colección Peña. Bartolomé Mitre escribe en un artículo sobre la imprenta jesuítica que antes de De Angelis el libro era desconocido (Mitre, 1896, p. 52-57). No es aquí el lugar de dar una apreciación de ese libro mayor de la historia de la imprenta argentina. Infelizmente no hay una edición en línea.

Analizamos a continuación, los manuscritos mencionados en el Apéndice. (ms. = manuscrito).

\section{Los manuscritos del Apéndice}

-Demo[n]stración clara, y evidente respuesta á las calumnias nuevamente inventadas contra los Relig[i]osos de la Compañía de Jesús de las Provincias del Paraguay, sobre el Catecismo, Oraciones y Doctrina Cristiana de la lengua guaraní, por el P. Díaz Taño. Autógrafo.

El texto del P. Francisco Díaz Taño (1593-1677) es conocido a través de la edición de Trelles de 1882. Díaz Taño fue rector del colegio de los jesuitas de Córdoba a partir de 1665 hasta su muerte, e hizo anteriormente en 1639 un viaje a Roma para obtener del papa Urbano VIII en 1639, la confirmación de la bula de Paulo II a favor de los indígenas, prohibiendo que se les esclavice. Tenemos su biografía en Jarque (1687, pp: 93-283). No sabemos el paradero del manuscrito original que fue publicado por Manuel Trelles, quien compró el manuscrito a De Angelis, en una de sus Revistas ${ }^{10}$. El texto fue probablemente escrito después de 1648 (Meliá, 1995).

\footnotetext{
${ }^{7}$ https://archive.org/details/jcbindigenous.

${ }^{8}$ La identificación de unos fragmentos del Martirologio, según la data verdadero primer impreso conservado de la primera imprenta jesuítica por Ernesto Maeder, no ha tenido mucha repercussion hasta hoy. El fragmento se encuentra en el Museo de Concepción vecino a Santa Cruz de la Sierra en Bolivia (Maeder, 2001, pp. 17-18, 46 y Maeder, 2008, p. 38).

9

La última edición facsimilar del libro infelizmente no está disponible al público, fue solo dado en donación a esponsores e instituciones. La introducción de Fernando Gil por enquanto está en línea: http://bibliotecadigital.uca.edu.ar/repositorio/investigacion/diferencia-temporal-eterno-juannieremberg.pdf. Las informaciones sobre la proveniencia del segundo ejemplar (colección Horácio Porcel, Buenos Aires) dados por Gil son en parte errados. Sobre la iconografia importante del libro ver esa introducción y Obermeier, 2006.

Revista de la Biblioteca Pública de Buenos Aires, tomo IV, Buenos Aires 1882, pp.17-80.
} 
Otra obra que viene atribuida al P. Francisco Díaz Taño es la Congregación y Iunta de personas Doctas, y peritas en la Lengua Guarani de los Indios de las Provincias del Paraguay... que mandó hacer el Ilustrissimo... Doctor Don Juan Alonso Ocon, Arzobispo de los Charcas en el Perú [Lima? 1661?]. Lo escribió para averiguar las calumnias que en aquellas Provincias se habían inventado contra los religiosos de la Compañia de Jesus, donde se discute la cualidad de las traducciones de oraciones en guaraní por los jesuitas, un tema mayor en el conflicto de los estos últimos y el obispo de Asunción, Bernardino de Cárdenas (1579-1668).

-Arte de la lengua guaraní, por el P. Blas Pretovio. (Anagrama de Pablo Restivo) Autógrafo, escrito en 1696.

De Angelis tenía un ejemplar del Vocabulario de la lengua guaraní, por el P. Antonio Ruiz de Montoya, extendido por el P. Pablo Restivo (Santa María la Mayor, 1722), listado también en el Apéndice.

Hay un ejemplar con el título del manuscrito en la Biblioteca de la Universidad de Granada: Arte de la lengua guaraní por el P. Blas Pretovio de la Compañía de Jesús. En el Vruguay. Año de 1696. [primera página] Sacado del Padre Antonio Ruiz y del Padre Alonso D’Aragona de los papeles del P. Sim.[on] Bandini y de otros. ${ }^{11}$ Como ese ejemplar proviene muy probablemente del Colegio de Córdoba, y nunca dejó esa colección, el que tenía De Angelis tiene que ser otro ejemplar. Sabemos que hay más ejemplares con ese título. ${ }^{12}$ La identificación del anagrama con Restivo parece ser de De Angelis que tenía también las ediciones de la imprenta jesuítica que Restivo viene mencionado. Ella fue después aceptada por todos los investigadores, sin cualquier crítica, lo que parece injustificado.

Tenemos varios ms. atribuidos al anagrama "Blas Pretorio". [la $\mathrm{r}$ puede fácilmente ser leído como v]. Uno se encuentra en el Museo Mitre con el título Compendio de los vocabulos mas usados de la lengua española y guaraní, sacados del vocabulario del Padre Blas Pretorio de la Compañía de Jesús (460 pág, 16 cm, 1729, Museo Mitre, $\mathrm{N}^{\circ}$. 4130). Otro ms. que tenemos del vocabulario de Restivo, después publicado en las imprentas jesuíticas el Vocabulario atribuido a Blas Pretorio, está encuadernado con la traducción en guaraní de la Conquista espiritual de Ruiz de Montoya (el ms. estuvo antes en Berlín, hoy en Cracovia).

\footnotetext{
${ }^{11}$ Recuperado de: http://hdl.handle.net/10481/17270.

Hay una foto de una carátula con ese título en Balmaceda (2004, p. 9), ilustración 4a. con referencia a la colección Mitre. Raúl Escandar de la Biblioteca Mitre, respondió en mail del 13.03.2012 que no está en el acervo del Museo. Por en cuanto Furlong, 1953, p. 52, menciona un ejemplar con ese título y data con ubicación en el Museo Mitre. Había robos en el Museo como vemos en el caso del ejemplar de la bibliografia de De Angelis con el Apéndice de De Angelis que había en ese Museo y que desapareció. Hay un ejemplar de ese Arte manuscrito que apareció en el mercado del libro antiguo en el año 2000 (www.lanacion.com.ar/17602). No sabemos donde está hoy. Agradecemos indicaciones sobre el paradero de ese manuscrito.

En la versión original disponible a través de busqueda en http://jbc.bj.uj.edu.pl/dlibra/: pág. 1-252v digital pág.509 con el nome de autor el pseudónimo Blas Pretorio, primero el Vocabulario, hasta pág.332 r. (pág. digital 667), datado en el colofone 1727, después la Conquista espiritual de Ruiz de Montoya en una de las dos versiones en guaraní que se conservan (datado al início 1733 en fin en el colofone 1737). La otra versión está en la Biblioteca Nacional de Río y fue editada por Almeida Nogueira en
} 
Para nosotros, esa identificación que está basada en la mención de Restivo en los impresos de la imprenta jesuítica de las doctrinas de los años 1720-1730 no tiene en cuenta el hecho que esa obra manuscrita era probablemente un trabajo colectivo de varios jesuitas, iniciado ya antes de la llegada del P. Restivo, tal como los otros manuscritos que llevan ese pseudónimo en el título. Restivo en 1696 solo había estado algunos años allí y no era probablemente tan experto en guaraní. Pensamos que la identificación de ese pseudónimo con otro jesuita -Pedro de Ramila- es posible (ver en bajo el ms. datado de las Phrases selectas, hoy en la colección del Museo Mitre). Esa identificación con un contribuyente mayor ciertamente, no prescinde de la colaboración de otros jesuitas.

\section{-Diario del desalojo de los Portugueses de la Colonia, en 1704. Manuscrito.}

Es cierto que el Diario lo compró Rafael Trelles, intelectual y coleccionista de Buenos Aires. La colección Trelles, primero propiedad de Rafael Trelles (1815-1880), a continuación, después de la muerte de su hermano Manuel Ricardo Trelles (1821 ó 1822-1893), fue comprada a los herederos por el ingeniero ferroviario argentino e historiador, Enrique Peña (1849-1924) en 1916. La colección de Peña llegó con objetos de arte en manos de su hija Elisa que al final de su vida cumplió el deseo de su padre de dejar la colección a una entidad pública (con pequeñas, más importantes excepciones como ese Diario del desalojo). Elisa donó la colección al Museo Udaondo en Luján, próximo a Buenos Aires. Su padre Enrique Peña fue en 1918 miembro de la Comisión Fundadora de este importante museo. Parte de esta donación fue la ya mencionada De la diferencia entre lo temporal y eterno del jesuita Nieremberg, traducido en guaraní y publicado en 1705. El manuscrito del Diario fue visto al tiempo de la donación al museo de Luján como de importancia menor y vendido. Hoy debe encontrarse en manos privadas.

Ese manuscrito de importancia mayor para la historia y lingüística rioplatense fue conocido a través de un artículo del P. Bartolomé Meliá (2002, pp. 217-222). Dentro del Proyecto Peky en Kiel hemos publicado una edición de ese precioso manuscrito junto con el Prof. Harald Thun y con Dr. Leonardo Cerno (2015).

Debido al hecho que el original no está disponible, nuestra edición y traducción está basada en la copia xerox en poder del Sr. Alejandro Larguía de Posadas, pariente de la familia Peña que también ha entregado una copia al P. Meliá. Nuestra edición con amplia introducción muestra el valor de ese texto, y referimos a este estudio para la historia complicada de la redacción del manuscrito que tiene cuenta de dos textos, una memoria simple, tal vez en guaraní y de otro, muy probablemente traducción de un texto mayor vertido del español en guaraní, y después fundido con la Memoria simple. Nuestra edición (Kiel: Westensee-Verlag 2015) lleva el título Lo que pasó en la guerra /Guarini hape tecocue tomado de un trecho del texto, resaltando que no se trata de un simple diario, sino de un texto muy elaborado que solo mantiene el carácter cronológico de un diario, transformándose en una obra literariamente trabajada. Para más pormenores ver los prefacios de nuestra edición. Una segunda versión corregida de nuestra edición fue publicada en Posadas: Flor del Desierto 2016.

Annaes da biblioteca nacional, Rio, texto tomo 6, 1878/79, Vocabulario (de la Conquista) tomo 7.1879/1880. En línea en las Anais de la Biblioteca nacional. http://objdigital.bn.br/acervo_digital/anais/anais.htm. Es datada 1754. Tiene el sello de la Real biblioteca. 
-Catecismos varios en lengua guaraní. Manuscrito en $4^{\circ}$.

Es una colección de varios manuscritos, todos catecismos, hoy en la colección de la Biblioteca Británica (British Library Add. 21262). En el catálogo están como Catecismos varios, y Exposiciones de la Doctrina Christiana, en Lengua Guarani. A proposito para hazerlas à los Indios, dispuestas por algunos Padres de la Compañía de Iesus. Y recogidas en la Doctrina de S. Nicolas. Año 1716, "Paper. Small Quarto". Nuestra pregunta al archivo de la biblioteca ha dado como data de adquisición según una nota en el ms. que era "purchased of Mr. H. Stevens, 12 April 1856". Un nativo de Vermont, Henry Stevens vivió en Londres alrededor de 1845, donde fue jefe de una librería especializada en libros con temas americanos, y buscó libros sobre las Américas para el British Museum y otras colecciones americanas en los Estados Unidos que estaban constituyéndose. ${ }^{14}$ Stevens fue un intermediario al cual De Angelis, siempre en necesidad de dinero, vendió ese ms.

-Frases selectas de la lengua guaraní. Manuscrito en $4^{\circ}$.

El ms. Se encuentra hoy en la colección del Museo Mitre. El catálogo lo menciona como Phrases selectas y modos de hablar escogidos y usados en la lengua guaraní, sacados del Thesoro escondido que compuso el venerable P. Antonio Ruiz de nuestra Compañía de Jesús para consuelo y alivio de los fervorosos misioneros, principiantes en la dicha lengua. Dedicación: fechada en San Francisco Javier, 27.09.1687. Tiene una página en francés, probablemente de uno de los posesores anteriores [Dictionnaire Espagnol-Guarani, composé en 1687, par le père Antonio Ruiz de la Compagnie de Jesús, Missions de Corrientes], que es errada. Otras notas de procedencia en el texto nos informan sobre sus propietarios anteriores a De Angelis: John Lelong [Le Long], agente colonial francés en La Plata y cónsul de Uruguay que vivió en la región cerca de 1840 a 1889, últimamente como cónsul en Rosario. ${ }^{15}$ Otra mención de procedencia en el libro es de un P[adre] Diego de Barjas [o Bayas] probablemente un sacerdote que lo tenía antes de Lelong. Hay una importante nota en la carátula: "Es del Pe. Ramila"; y al pié "De San Xavier", lugar mencionado en el Prólogo. Se trata del jesuita Pedro de Ramila (Buenos Aires, 1678 - Santa María de Fe, 1748) (Storni, 1980, p. 232). La gra-fía de esa nota es de la época de la redacción. Una cuestión fundamental es si ese P. Ramila era el autor del texto o el propietario del manuscrito. Para mí es el principal autor, siempre considerando otras contribuciones. Hay pocas menciones de nombres de jesuitas como dueño de manuscritos o libros en los ms. de la época. La mayor parte de los jesuitas tenían tal vez algunos libros privados o manuscritos, más no escribieron por modestia su nombre y esos libros eran ciertamente pocos. Solo los provinciales tenían a su disposición una pequeña librería de libros que eran prestados por la biblioteca jesuítica de Córdoba y puestos a su disposición o del Padre Superior. ${ }^{16}$ Tampoco en los sermonarios

\footnotetext{
${ }^{14}$ Los textos en Londres son editados Catecismos vários, São Paulo: Universidade de São Paulo, Facultad de Filosofia, Ciências e Letras, Boletim, 6 vols. 1952/56.

12 Franz Obermeier. El Apéndice de la Colección de obras impresas y manuscritas... 3-27. 
rioplatenses manuscritos de la época que estamos investigando al momento (no publicado) no hay menciones de un nombre de jesuita ni como propietario, ni como autor o copiador.

Sabemos muy poco de Padre Ramila. ${ }^{17}$ Para mí el pseudónimo "Blas Pretorio" de los manuscritos (no hay un jesuita de tal nombre o de un nombre semejante) puede bien ser un anagrama de Padre Ramila.

\section{Pedro de Ramila}

\section{Blas Pretorio}

Eso explicaría también que tales textos ya aparecen con datas en que el P. Restivo probablemente no sabía tan bien el guaraní. Las frases selectas son datadas, dedicadas a los "fervorosos y Apostólicos Padres misioneros", San Francisco Xavier, 27 September 1687. Pablo Restivo no estaba en el Rio de la Plata en $1687 .^{18}$ Sobre el contenido del texto referimos al nuevo artículo de Chamorro (2014). Es una interesante actualización de la obra lingüística de Ruiz de Montoya (Tesoro de la lengua guaraní, Madrid: Sánchez 1639) que considera los cambios lingüísticos casi 50 años después de las obras de Montoya.

Hace poco los investigadores del grupo francés Langas, han encontrado otro ejemplar de las Phrases selectas (con título al dorso en francés: Frases en guaraní, como el de los Diálogos es: Diálogos en guaraní, lo que muestra que provienen de una colección) en la biblioteca del Museo Udaondo de Luján junto con otra versión del manuscrito de los Diálogos (Adoue, et. al., 2015 ver nuestro párrafo sobre ese manuscrito en seguida que es también de De Angelis). Pensamos que la explicación más probable aquí es que De Angelis tenía dos ejemplares de ese manuscrito también como tenía dos manuscritos de los Diálogos, y que los usaba para poder leer ese manuscrito difícil, tal vez habiendo encontrado los dos documentos en una misma colección y haciéndolos encuadernar nuevamente con encuadernaciones semejantes.

\section{-Compendio de la lengua guaraní. Manuscrito en 12.}

Compendio de los vocabulos más usados de la lengua española y guaraní, sacados del vocabulario del Padre Blas Pretorio de la Compañía de Jesús, 1729.

Se encuentra en la colección Mitre, catálogo, Nº 4130. Hay una descripción detallada en Documenta iesuitica, Octubre 2000, pág. 2. Tiene 234 pág. Mitre cota 14.1.14. Nueva encuadernación.

nota sobre los libros de esa biblioteca en el cuarto del Padre Superior: "De lengua guarani en $8^{\circ}$ : 1054 , en $4^{\circ} 87$, en fólio: 2,1143 ", números enormes cuando pensamos al tiraje de libros especializados en lenguas indígenas en la época. Sobre el probable tiraje de los libros de la imprenta jesuítica ver Obermeier, 2017 (en prensa).

\footnotetext{
${ }^{17}$ Viene mencionado en Telesca (2006, p. 78) o Furlong (1962, p. 138).
}

${ }^{18}$ La atribución del texto a Restivo por Meliá, 1992, p. 93 no tiene fundamentos y muestra la poca investigación sobre los manuscritos jesuíticos que se centra en las figuras mayores de Ruiz de Montoya y Pablo Restivo (Obermeier y Cerno, 2013, pp. 33-56). 
-Diálogos en lengua guaraní. Manuscrito en 12.

Se trata de un ms. que De Angelis vendió a su amigo personal, el embajador prusiano Friedrich von Gülich, embajador del Zollverein después prusiano que por eso es considerado el primer embajador alemán en la región. Vamos a dar los argumentos en favor de nuestra hipótesis en seguida.

Hay dos versiones de ese texto que vamos a presentar. La identificación del ms. de Gülich [G.] de Luján [L.] con el del Apéndice es también posible porque no hay otro ms. que usa la forma de "Diálogos" en el ámbito de textos del temporal. Los textos religiosos del Apéndice están siempre alistados con mención del género (Catecismo, etc.).

Hemos descubierto una copia manuscrita de G. hecha por intermedio del lingüista alemán Julius Platzmann a pedido de Carl Henning para la colección del Emperador del Brasil. El emperador quería hacerlo traducir por el especialista del guaraní Almeida Nogueira, que murió antes y la copia acabó en la colección de un lingüista estadounidense, Daniel Brinton (1837-1899) que la ha dado toda en legado a la Penn Library en Philadelphia En el Proyecto Peky preparamos una edición del texto de Philadelphia. ${ }^{19}$ El original quedó en manos de la familia de los Gülich y se destruyó durante los bombardeos de la Segunda Guerra Mundial en Wiesbaden. Investigadores franceses (grupo Langas) han encontrado otra versión del Gülich en la biblioteca del Museo Udaondo de Luján. Según ellos, el ms. de Luján contiene casi 200 páginas más que el ms. Gülich y pasajes idénticos con el ejemplar de Gülich hoy en Philadelphia. El Gülich contiene también pasajes que hacen falta en el texto de Luján. Hay varias posibilidades sobre la procedencia de esas dos versiones del ms. de Diálogos en guaraní al momento actual de la investigación.

El hecho de tener dos variantes de un mismo texto como copias de una fuente desconocida que podemos llamar $\mathrm{G} / \mathrm{L}^{*}$ no es algo que puede extrañar. Tales textos existieron en varias versiones, y la del Gülich es tal vez una que circuló en las reducciones y era adaptada por un copiador a su propio dominio lingüístico y a las necesidades de su trabajo en una reducción.

Es claro que muchos textos de la colección De Angelis llegaron a través de la colección de los Trelles, más tarde de Enrique Peña por donación de su hija Elisa al Museo de Luján en 1954. Ese manuscrito de Luján es el de De Angelis porque contiene un sello de la "Donación Peña". ¿Más de dónde viene el ms. de Gülich? ¿Sería de proveniencia desconocida y no de la colección De Angelis?. Gülich podría haber encontrado el manuscrito en Asunción o en uno de sus múltiples viajes a la zona rioplatense. Existe un ms. del Vocabulario de Restivo que Gülich ha encontrado en Asunción y que antes aparecía como donación de Gülich a la Biblioteca de Berlín (Staatsbibliothek), hoy está en Cracovia. Ese último ms. tampoco es de De Angelis y no se encuentra en el Apéndice, Gülich anota en la portada que lo ha comprado en Asunción.

Hay aunque otra posibilidad que nos parece la más probable sin poderse probar con documentos. De Angelis no tenía solo una, sino dos versiones del manuscrito. Conservó el manuscrito más completo que era L según el tamaño para su colección y lo ha vendido a Trelles, solo cuando se decidió a vender la colección entera. Ese ms. llegó como sabemos después a través de dos colecciones en Luján.

${ }^{19}$ Sobre la proveniencia y el manuscrito ver Obermeier (2011, pp. 167-183).

14 Franz Obermeier. El Apéndice de la Colección de obras impresas y manuscritas... 3-27. 
El otro ms. menos completo, De Angelis lo vendió a su amigo Gülich porque sabía de un examen rápido que era probablemente un extracto de sus Diálogos más "completos" o por lo menos muy semejante.

El Apéndice no menciona el segundo ms., lo que es lógico porque el Apéndice no es -como tenemos que recordar- una descripción bibliográfica de una colección, sino un pequeño catálogo de venta como toda esa bibliografía de De Angelis. Solo se puede vender lo que todavía se tiene o no se tiene ya prometido a un amigo como Gülich.

Sin embargo, el Apéndice menciona el único otro manuscrito que De Angelis ha vendido suelto al extranjero, los Catecismos hoy en la British Library. Tenemos que preguntarnos porque es el caso para ese manuscrito y no para los Dialogos. Puede ser debido a la data de la venta de ese ms. a Londres. Nuestra pregunta al archivo de la biblioteca ha dado como data de adquisición según una nota en el ms. que era "purchased of Mr H. Stevens, 12 April 1856”. Y porqué De Angelis no conservó esos dos ms, los catecismos y el Gülich en la versión G.? Pienso que hay también una razón ligada al contenido que lo explica: De Angelis ya tenía tres catecismos impresos en guaraní, todos los que hay en la época colonial o poco después en impreso: Montoya, Yapuguay y Bernal. Claramente podría tener visto que la versión de eses ms. no era idéntica con esos impresos, más para un texto tan formalizado como lo son los Catecismos, una u otra versión en ms. no le otorgaba dar nuevo saber sobre un tema, que con sus intereses mayormente históricos y lingüísticos le interesaba menos que los contenidos de sus otros ms.

Si aceptamos la hipótesis que también el ms. G. era de la propiedad de De Angelis, podemos identificar una estrategia de venta: en un primer periodo de venta únicamente ha vendido los textos que le tenía duplicados (sin serlo exactamente para nuestro juicio hoy), y ha rigurosamente conservado la otra parte de la colección etnolingüística en conjunto, hasta la venta de todo a solo dos personas: Mitre y Trelles. Inició con la venta de eses duplos: la versión de los Diálogos de Gülich tal vez en el inicio de 1856 y los Catecismos varios antes el 12 de abril 1856 cuando entraron en la British Library. En el caso de las Phases selectas era fácil más tarde, porque podía vender uno de sus dos ejemplares a Mitre (hoy en el Museo Mitre) y el otro a Trelles junto con su versión más completa de los Diálogos. Eso no es sin una lógica de uso y práctica para un personaje tan previsor y premeditado como lo era Angelis. El valor de la colección etnolingüística en total no disminuyó con esas ventas sueltas. Perdía con la venta de un ms. de los Diálogos al Gülich y la otra de los Catecismos varios a menos "valor material" para una investigación futura en su propia colección que posiblemente conservó por lo menos para un cierto tiempo el cuerpo de su colección que lo tenía arraigado a su corazón. Después a una data antes de junio 1856 (su Carta a Tomás Guido, el 18. Junio 1856 menciona que ni siquiera tenía más un vocabulario de guaraní), más probablemente en mayo 1856 vendió el resto de su colección a solo dos personas, Mitre y Trelles.

No es aquí posible de presentar pormenores de eses Diálogos. Tenemos que remitir el lector interesado a nuestra edición del ms Gülich prevista (Harald, et.al.).

El texto es sobre la vida diaria, casi una "enciclopedia del quotidiano" con normas de comportamiento en las reducciones, puniciones y instrucción. El cuadro de Diálogos entre un indígena y un jesuita sirve a jóvenes misioneros a aplicar en un uso pragmático correcto y con carácter de modelo la explicación de esas normas. Por eso es un de los textos mayores del temporal en la región. 
IHS. Antiguos Jesuitas en Iberoamérica ISSN: 2314-3908

-Notas al Catecismo en español y guaraní, publicado por Fray José Bernal, P. Provincial de San Francisco de Buenos Aires. Manuscrito.

El texto al cual se refieren esas notas estaba también en posesión de De Angelis y viene mencionado en el Apéndice. Se trata del Catecismo de doctrina christiana en guaraní y Castellano, para el uso de las curas doctrineras de indios de las naciones guaraníes de las Provincias del Paraguay, Pueblos de Misiones del Uruguay y Paraná, Santa Cruz de la Sierra, naciones de Chiquitos, Matagayos y Provincias de San Pablo de los Portugueses, e instrucción de los mismos pueblos, En la Real Imprenta de los Niños expósitos 1800.

El franciscano José Bernal era de los sacerdotes que se ocuparon de los pueblos indígenas después del exilio de los jesuitas. No sabemos que eran esas notas, tal vez notas posteriores de algunas páginas hechas por un propietario de uno de los raros impresos. Hay tales notas en el ejemplar de la Biblioteca Hispánica (AECID) de Madrid. La biblioteca ha respondido a mi pregunta que, se trata de un libro, el "Catecismo de doctrina christiana..." y un documento relacionado con ese libro, que se titula: "Notas al catecismo en castellano y guaraní que ha dado a luz sin ser su autor el R. P. provincial de San Franco. De Buenos Ayres Fr. José Bernal". Este ejemplar de Madrid es un manuscrito con múltiples tachaduras de una posible censura. Es un cuadernillo de 2 hojas, y están escritas 3 páginas, y la última está en blanco. El libro impreso mide 14,3 cm, y no existen marcas de anteriores propietarios. El cuadernillo manuscrito mide 30,8 cm y no existen marcas de anteriores propietarios.

Por causa de la ausencia de marcas de anteriores propietarios no nos es posible de identificar ese manuscrito con el de De Angelis, mas es probable que sea el suyo. Hay una mención de tales notas en la literatura en Juan María Gutiérrez (1866; sobre Bernal No 102, pp. 379-382). En página 382 se refiere al Apéndice de De Angelis y a las Notas de esa colección. El artículo de Gutiérrez es sobre la primera imprenta laica de Buenos Aires, llamada Niños Expósitos fundada en 1780 y más tarde Imprenta del Estado.

El manuscrito original del impreso está con el título Cathesismo de la lengua Guarany y Castellana para la instrucción de los Misioneros Curas de Yndios de las provincias del Paraguay, Santa Cruz de la Sierra, y naciones de Chiquitos, y los pueblos de las Misiones Uruguay y Para, y pueblos del Chaco, y provincia de San Pablo de los Portugueses (1790) en la Bibliotheca Real de Madrid.

-Oficios en lengua guaraní, con las respectivas traducciones en español. Manuscrito en fol.

Hoy en el museo Mitre, en nueva encuadernación hecha por Mitre con el título Misiones del Uruguay 1758-1783, catálogo No 4502, según los Documenta iesuitica cota 14.8.18. Hay varias descripciones de esos textos que son órdenes del gobernador Pedro de Cevallos, la mayoría son de 1761 (Neumann, 2005). ${ }^{20}$

\footnotetext{
${ }^{20}$ Los documentos de 1772 como No 118 und 119, una lista de Indios en un recibo de 1766; la Lista de 78 indios de San Boja y Santo Tomé que acompañaron a un resguardo, solicitados por Martín de Altolaguirre, en 7 de julio de 1766 no viene mencionada. Una inscripción en una cruz es listada como $\mathrm{N}^{\circ} 49$, pero con referencia a otra fuente el Diario de los Demarcadores durante el Tratado de límites en la Bibli-
}

16 Franz Obermeier. El Apéndice de la Colección de obras impresas y manuscritas ... 3-27. 
-Arte breve de la lengua guaraní. Manuscrito en 12.

Del manuscrito de Angelis que hoy se encuentra en el Museo Mitre, hay una copia en el Colegio del Salvador. Ese último ejemplar ha sido editado por B. Meliá (1979, pp. 23-61 y 1980). Tiene tres partes Breve Introduccion para aprender la lengua guaraní por el $\mathrm{P}$. Alonso de Aragona 37 págs. +1 pág. en blanco. Confessonario. 15 págs. Cathecismo que se resa en la Yglesia. 21 págs.

-Confesionario de la lengua guaraní. Manuscrito en 12.

Museo Mitre, catálogo 4208, cota 14.2.27. Descripción en los Documenta iesuitica Octubre 2000, $\mathrm{N}^{\circ} 0122$.

-Texto de la doctrina cristiana, por el P. Bolaños. Manuscrito en 12.

Es conocido que el franciscano fray Luis de Bolaños (Marchena (Sevilla, 1539 (?)- Buenos Aires, 1629) hizo una traducción de las oraciones en guaraní en seguida del célebre Tercero Concilio de Lima que ordenó la predicación en las lenguas indígenas de la región. ${ }^{21}$

En el Museo Mitre hay de Luis de Bolaños, Testimonio del catechismo y oraciones en la lengua guaraní del Paraguay [con notas del Dr. Julián de Leyva]. Mitre catá$\log \mathrm{N}^{\circ} 4497$.

Julián de Leyva o Leiva (1749-1818) era uno de los más conocidos críticos de la Revolución de Mayo. Tenía una amplia colección de libros. Puede ser que Angelis tenía comprado el ms. de esa colección.

-Explicación de la doctrina cristiana. Manuscrito en 12.

Identificación dudosa.

Tal vez el ejemplar en el catálogo Mitre, No 2908 con el título El TEXTO de la doctrina christiana en lengua guaraní y castellana, [128 pág.]. - $21 \mathrm{~cm}$, (ejemplar manuscrito): Con anotaciones de Mitre, Bartolomé, referidas a la publicación de la obra en Madrid, 1740. [Sic para 1640].

Cardozo (1959, pp. 243-244) describe ese ejemplar. Hay una nota de Mitre que menciona que era donación de un cierto Juan Pablo Linch "quien lo encontró en la Asunción". Pablo Linch es de cierto el presbítero Don José Pablo Linch mencionado varias veces en fuentes de la época. En ese caso no sería de la colección de Angelis, si

oteca nacional de Rio.Ver también Meliá (2010), el convolto de la colección Mitre en Documenta iesuiti$c a \mathrm{n}^{\mathrm{o}} 24$, octubre 2000 , documento 0125 .

${ }^{21}$ Sobre su biografia ver Heras (1991, pp. 203-206).

${ }^{22}$ La nota viene citada en Furlong (1964, p. 164). 
IHS. Antiguos Jesuitas en Iberoamérica ISSN: 2314-3908

se hay de suponer una donación hecha a Mitre. Descripción del ejemplar Mitre en Documenta iesuitica Octubre 2000, $\mathrm{N}^{\mathrm{o}} 0122$.

El texto de Angelis puede ser un extracto del gran número de manuscritos de catecismos que había en las reducciones.

[En la sección de lenguas chilenas]

-Doctrina cristiana. Manuscrito en 12.

Identificación probable.

La dificultad radica en el hecho de que "chileno" aquí pueden ser diferentes idiomas. Solo una cosa es cierta: Angelis tiene en una sección anterior en sus libros en las lenguas aymará y quechua por eso tiene que tratar de otra lengua. Hay documentos coloniales sobre mapundungun ("mapuche" en la época) y allentiac pero esos últimos se conservan solo en impresos. El manuscrito podría también ser una copia de las partes correspondientes de Luis Valdivia, Doctrina Christiana en mapundungun. Pero el más probable es otro.

Anteriormente, el Apéndice de esa sección justamente alista la primera obra sobre el mapundungun, el libro del jesuita Andrés Febres (1734-1790), Arte de la lengua en general del Reyno de Chile parecido en Lima en 1765. Tal vez este manuscrito de la Doctrina Christiana era una copia de la parte que hizo falta a su edición del Febres. De Angelis menciona sólo el Arte y el vocabulario contenidos en su ejemplar. Era usual que se completaron libros deficientes de páginas o algunas partes con textos a mano. El ejemplar del Museo Mitre de la obra de Febrés es muy probablemente el que menciona De Angelis y que Mitre compró. Está incompleto (catálogo Mitre № 4163, faltan las pág. 1-414, eso es el Arte y la Doctrina, solo hay el Vocabulario). De Angelis hizo copiar por lo menos la Doctrina de otro ejemplar tal vez en una biblioteca europea.

Es menos probable que ese ms. de De Angelis era una copia de Luis Valdivias (1607) porque de ese texto en el siglo XIX solo se conocía el ejemplar impreso de la Biblioteca Nacional de Madrid, después en el fin del siglo, base de la primera reedición que apareció como Doctrina Cristiana y catecismo, con un confesionario, arte y vocabulario breves en lengua allentiac por el Padre Luis de Valdivia con una "Reseña de la vida y obras del autor" por José Toribio Medina, Sevilla: Rasco 1894. Esta edición también la tuvo Mitre que publicó sobre el tema un libro suyo (Valdivias, 1607) ${ }^{23}$. La lengua allentiac también fue hablada por indígenas de origen cuyano, que para trabajo forzado pasaron a Santiago de Chile. Sin embargo, no existe ningún rastro de un manuscrito en allentiac.

Otro ejemplo de esas copias de partes de manuscritos hechas en el siglo XIX es un Confessionario breve en la lengua del Reyno de Chile (1868) que se encuentra a partir de 1868 en la colección de la John Carter Brown Library. Una nota explica que 1868

\footnotetext{
23 Mitre publicó sobre esa lengua que consideró argentina su libro Lenguas americanas, estudio bibliográfico-lingüistico de las obras del P. Louis de Valdivia sobre el Araucano y el Allentiak, con un vocabulario razonado del Allentiak, primero en la Revista del Museo de La Plata, después como libro en La Plata 1894. La lengua allentiac se habló en la región del Nuevo Cuyo de la Argentina. Trabajadores de allí fueron deportado para trabajos forzados en Santiago de Chile donde Valdivia ha documentado la lengua. Otra designación de dialectos de esa lengua son huarpe o millcayac.
}

18 Franz Obermeier. El Apéndice de la Colección de obras impresas y manuscritas... 3-27. 
es la data de la adquisición del manuscrito, un Terminus ante quem. La última página blanca lleva un pequeño Ex-Libris del vendedor de libros antiguos de Paris Maisonnneuve, especializado en Americana.

El lingüista alemán Julius Platzmann (ver encima sobre el Ms de Diálogos en guaraní) trabajó también con copias de impresos para preparar sus ediciones facsimilares. Las gramáticas impresas de lenguas indígenas eran en ese tiempo muy raras y de algunos solo había uno o dos ejemplares, y el paradero de otros no era conocido antes de la época de catálogos impresos o en línea.

\section{La venta de la colección etnolingüística de De Angelis}

La venta de la colección etnolingüística de Angelis no está documentada. Tenemos en el ms de los catecismos varios hoy en la British Library como data de adquisición una nota ya mencionada que era "purchased of Mr H Stevens, 12 April 1856". La data es un terminus ante quem que vale por lo menos para ese manuscrito, mas muestra también que De Angelis en ese tiempo tenía la intención de vender su colección etnolingüística. Puede ser deducido de un pasaje de carta de De Angelis que pidió un diccionario de guaraní a un amigo, el General Tomás Guido, el 18. Junio 1856 "Me he quedado sin ninguna [obra lingüística] por haber vendido aquí, no al Brasil, mi colección" (Sabor, 1995, p. 192) ${ }^{24}$. Esa data es otro terminus ante quem que vale para toda la colección

La motivación de De Angelis de vender esa parte, no al Brasil sino en Buenos Aires, era porque era duplicada. No estaba contento con la suma de dinero (8.000 pesos en vez de los 12.000 que esperaba) que el Brasil había pagado para su única colección de manuscritos e impresos históricos. Sabía ciertamente que Pedro II se interesaba por la etnolingüística, porque consideraba las lenguas tupi-guaraní (el tupinamba y el guaraní) como parte de la historia del Brasil e inspiró ediciones de textos como la traducción y edición de la Conquista espiritual de Montoya en guaraní por Almeida Nogueira, en los Annaes de la Biblioteca Nacional (tomo 6, 1878/79, y tomo 7.1879/1880). Pedro II por eso habría ciertamente comprado también esa parte única de la colección De Angelis. Más era De Angelis que no quería venderla a Pedro II.

Su estrategia de venta no es solo debida a intereses pecuniarios, más también al hecho que De Angelis sabía del valor único de los ms. y libros en lenguas indígenas para sus investigaciones. Esa idea viene formulada en una carta de De Angelis a Rafael Trelles, uno de los interesados en una compra. Él escribe a Rafael Trelles el 3.5.1856: "El sentimiento que naturalmente tengo de separarme de la parte más preciosa de mi biblioteca queda en gran parte atemperado por la idea de que los nuevos poseedores son personas inteligentes, y que saben apreciarla".

Por eso la razón tal vez decisiva para De Angelis de no venderla a Pedro II era (también anticipando críticas que fueran después hechas a él por la venta de tan valiosa

\footnotetext{
${ }^{24}$ Ver la cita en Becú y Torre Revello (1941, p. XLIX-LII).

${ }^{25}$ La carta de una colección privada no especificada Recuperado de Libreria Fernáandez Blanco (ed.), Un incunable rioplatense, 2004, pág. 39-40, aquí pág. 39. Nos ayuda también a datar la venta porque menciona que Trelles examinó en data anterior los libros que le interesaron.
} 
documentación fuera de la zona rioplatense) que quería continuar él mismo en trabajar sobre el guaraní, como lo prueba su Diccionario manuscrito del guaraní. ${ }^{26}$

Con que era para él menester dejar esa parte de su colección en mano de personas fiables y viviendo en Buenos Aires donde De Angelis volvió en 1856 cuando no era más persona grata y su soporte a Rosas era olvidado. Así Angelis podía tener también libre acceso a los originales. Más su muerte en 1859 impidió que pudiera contar con el tiempo para escribir una obra lingüística mayor. Las investigaciones etnolingüísticas de Angelis documentadas solo en notas y manuscritos en el AGN todavía están a investigar.

\section{Conclusión}

Todo parece indicar que De Angelis deliberadamente ha retenido la parte etnolingüística de su colección, y por varias razones. La consideró la más valiosa, la que quiso mantener para futuras investigaciones en Buenos Aires o en sus proximidades, y por ello, solo vender a coleccionistas amigos y fiables como Rafael Trelles y Bartolomé Mitre. De hecho era ciertamente la colección más valiosa de gramáticas jesuíticas coloniales en esa época en manos privadas. Es casi desconocido hoy que Angelis haya hecho su propia investigación sobre lenguas indígenas, aunque dejó un Diccionario guaraní manuscrito que hemos redescubierto en el AGN de Buenos Aires. Los manuscritos e impresos en lenguas indígenas de su colección fueron vendidos por él, aproximadamente entre abril y mayo de 1856 en Buenos Aires. La mayor parte fue adquirida por Bartolomé Mitre, y hoy consta en la colección del Museo Mitre en Buenos Aires, antigua residencia de Mitre. Algunos libros y manuscritos fueron vendidos al coleccionista porteño Rafael Trelles, esa colección pasó en las manos de Enrique Peña en 1916 y en parte después al Museo de Luján con notables excepciones como el Diario del desalojo. Piezas individuales las vendió Angelis mas solo cuando las consideró duplas o de menor importancia a Friedrich von Gülich (tal vez en el inicio de 1856), y una colección de catecismos manuscritos en guaraní al Museo Británico, hoy la British Library, a través de un intermediario antes de abril de 1856.

La venta de la colección fue, por supuesto, de acuerdo con los intereses de los coleccionistas, pero se ve bien que Bartolomé Mitre estaba sobre todo interesado en gramáticas y manuscritos fácilmente utilizables por personas que no sabían guaraní. Tal vez ya pensó en publicar sobre lenguas indígenas o hacer una colección de materiales como hizo después. Los textos íntegramente en guaraní le interesaban menos, porque había pocas personas en Buenos Aires que en el siglo XIX podían leer textos semejantes, dada la ausencia de una filología guaraní o de conocimientos filológico-históricos sobre el guaraní en la época.

Solo mucho tiempo después, a la muerte de Trelles, Mitre hizo una oferta para comprar el Nieremberg en guaraní de sus herederos, como sabemos de su artículo sobre las Origenes de la imprenta jesuitica, mas no lo consiguió. Trelles (o Gülich, por el manuscrito Diálogos, versión G) o el British Museum adquirieron los manuscritos en guaraní más como ejemplares raros o curiosidades de una tradición del guaraní escrito que con un interés en una publicación inmediata o traducción. Pero Gülich permitió hacer

${ }^{26}$ AGN, Legajo Andrés Lamas 22 y 23, en $N^{\circ}$ 23: Estudios y Vocabulario de la lengua guarani por Pedro de Angelis.

20 Franz Obermeier. El Apéndice de la Colección de obras impresas y manuscritas... 3-27. 
una copia de su manuscrito cuando se lo pidió el Emperador Pedro II, a través del intermediario de Karl Friedrich Henning, su profesor de lenguas, a Julius Platzmann. La venta de una colección de catecismos a la Biblioteca del British Museum se debe seguramente a la intención de esa biblioteca de contar con una colección universal.

El Apéndice de De Angelis y nuestra investigación nos dan no solo importante saber sobre la procedencia de manuscritos mayores escritos en las reducciones jesuíticas rioplatenses, sino también una luz sobre la importancia de una parte casi desconocida de su colección. Tal vez puede también cambiar un poco la imagen negativa sobre De Angelis en Argentina debido a la venta de su biblioteca al Brasil. Era muy cuidadoso con la parte etnolingüística de su colección que quería conservar en manos privadas de amigos coleccionistas de Buenos Aires como parte del patrimonio nacional argentino.

\section{Referencias}

Adoue, C., Boidin, C. y Orantin, M. (2015). Les Diálogos en guaraní, un manuscrit inédit des réductions jesuites du Paraguay (XVIII siècle). Novos Mundos, Nouveaux mondes, https://nuevomundo.revues.org/.

AGN. Archivo General de la Nación Argentina (Buenos Aires).

Balmaceda Abrate, J. C. (2004). El origen de la imprenta argentina: introducción al estudio del incunable guaraní impreso en Loreto. En Isabel I y la imprenta... Madrid. Recuperado de http://www.cahip.org/origen_imprenta_argentina.pdf.

Becú, T, y Torre Revello, J. (1941). La colección de Documentos de Pedro de Angelis y el diario de Diego de Alvear, Buenos Aires.

Brabo, F. J. (ed.) (1872a). Colección de documentos relativos a la expulsión de los jesuitas de la república Argentina y del Paraguay em el reinado de Carlos III. Introducción y notas de D. Francisco Javier Brabo, Madrid: Imp. y Esterotipia de W. Rivadeneyra. Recuperado de https://archive.org.

(ed.) (1872b). Inventarios de los bienes hallados en los pueblos de Misiones de ambas márgenes del Uruguay y Paraná, al tiempo de la expulsión y ocupación de temporalidades de los jesuitas. Madrid: Imp. y Esterotipia de W. Rivadeneyra. Recuperado de http://bibliotecadigital.aecid.es/bibliodig/es/consulta/resultados_ocr.cmd

Brinton, D. G. (1891). The American race. A linguistic classification and ethnographic description of the native tribes of North and South America. New York: Hodges.

Campbell, L. (ed.) (2012). The indigenous languages of South America. A comprehensive guide. (The world of linguistics, 2) Berlin: de Gruyter Mouton.

Cardozo. E. (1959). Historiografia paraguaya, México.

Censabella, M. (1999). Las lenguas indígenas de la Argentina. Una mirada actual. Buenos Aires: Eudeba.

Cerno, L. (2013). El guaraní correntino. Fonología, gramática, textos. (Mikroglottika, 6) Frankfurt am Main: Lang. Recuperado de http://www.etnolinguistica.org/.

Cerno, L. y Obermeier, F. (2013). Nuevos aportes de la lingüística para la investigación de documentos jesuíticos de los siglos XVII y XVIII. Folia Histórica del Nor- 
IHS. Antiguos Jesuitas en Iberoamérica ISSN: 2314-3908

deste, Resistencia (Chaco), 21.2013.33-56. Recuperado de https://www.academia.edu/.

Chamorro, G. (2014). Phrases selectas, un diccionario manuscrito castellano-guaraní anónimo. Corpus Vol. 4, No 2. Recuperado de http://corpusarchivos.revues.org/.

Cortesão, J. (1951-1969). Manuscritos da coleção de Angelis. Rio de Janeiro: Biblioteca Nacional. Recuperado de www.pucrs.br/delfos/?p=angelis

Créqui-Montfort, G. de y Rivet, P. (eds.) (1951-1956). Bibliographie des langues aymará et kičua. (Travaux et mémoires de l'Institut d'Ethnologie, Université de Paris; ...). 4 vol. Paris: Inst. d'Éthnologie [documentos de 1540 hasta 1955].

Cutolo, V. (1975). Nuevo Diccionario biografico argentinos. Buenos Aires, tomo IV, usado a través la base de dados del World Biographical Index.

De Angelis, P. (1836-1837). Colección de obras y documentos relativos a la história antigua y moderna de las Provincias del Río de la Plata. Buenos Aires, Argentina: Imprenta del Estado, 6 volúmenes.

(1853). Colección de obras impresas y manuscritas que tratan principalmente del Río de la Plata (Sin lugar, sin impresor) en línea: http://www.cervantesvirtual.com/ y http://www.biblioteca.org.ar/libros/300749.pdf. Apéndice a esa bibliografia (3 pag., sin lugar, sin impresor, antes de 1856). Ejemplar de la Biblioteca de la Universidad Nacional de La Plata, Colección Farini. Base de la transcripción en ese artículo y probablemente el único ejemplar que existe todavia. Biblioteca Digital Curt Nimuendajú. Línguas e culturas indígenas sul-americanas, Repositorum: http://www.etnolinguistica.org/.

Documenta iesuitica. Revista editada por Luis Pérez-Cuesta.

Fabre, A. (s/f). Diccionario etnolingüístico y guía bibliográfica de los pueblos indígenas sudamericanos. Publicación dinámica: http://www.ling.fi/DICCIONARIO.htm.

Fondo antiguo de la Compañía de Jesús en Argentina. Recuperado de https://esla.facebook.com/Laboratorio-Fondo-Antiguo-494967853956583/ Sin catálogo de la colección.

Furlong Cárdiff SJ, G. (1962), Misiones y sus pueblos de Guaranies. Buenos Aires: Impr. Balmes.

Furlong SJ, G. (1964), Antonio Ruiz de Montoya y su Carta a Comental (1645). Buenos Aires: Ed. Theoria 1964.

(1953). La imprenta en las reducciones del Paraguay, 1700 - 1727. La imprenta en Córdoba, 1765 - 1767. La imprenta en Buenos Aires, 1780 - 1784, [La imprenta en Montevideo, 1807 - 1810]. (Historia y bibliografía de las primeras imprentas rioplatenses, 1700 - 1850: misiones del Paraguay, Argentina, Uruguay, 1) Buenos Aires: Guaranía.

Gutiérrez, Juan María (1866). Bibliografia de La Primera Imprenta de Buenos Aires Buenos Aires. Buenos Aires: Impr. de Mayo (en partes en separada antes en la Revista de Buenos Aires, ed. por Ernesto Quesada, junio 1865-agosto 1866 aquí la Continuación 1866, pp. 369-407). 
Harald Thun, Leonardo Cerno, Franz Obermeier (eds.) (Inédito). Âng tobeete acôy teco cò mombürăagui - Dejad ahora aquellas costumbres ancestrales. Manual anónimo en guaraní de la gestión de las Reducciones (inicio del siglo XVIII, Manuscrito Gülich), edición crítica con reproducción facsimilar del original, traducción al castellano, introducción y notas.

Heras OFM, J (1991). Fray Luis de Bolaños. Iniciador de las «reducciones» de Paraguay, Recuperado de R. Ballán, Misioneros de la primera hora. Grandes evangelizadores del Nuevo Mundo. Lima, 203-206 y http://www.franciscanos.org/enciclopedia/lbolanos.html.

Hervás y Panduro SJ, L. (1800-1805). Catálogo de las lenguas de las naciones conocidas y numeracion, division, y clases de estas según la diversidad de sus idiomas $y$ dialectos. 6 vol. Madrid: Ranz 1800-1805. Recuperado de http://www.liburuklik.euskadi.net/handle/10771/24570.

Jarque, F. (1687), Insignes missioneros de la Compañia de Jesvs en la provincia del Paraguay, Estado presente de sus missiones en Tucuman, Paraguay, y Rio de la Plata, que comprehende su distrito. Por el doct. d. Francisco Xarqve, ... Que remite, y consagra à los religiosos operarios, y apostolicos missioneros, que al presente prosiguen sus heroycas empressas, por mano del ... padre Thirso Goncalez de Santalla, Preposito General, y atlante de las missiones que por todo el orbe exercita la religion amplissima de la Compañia de Jesvs. Pamplona: Micon 1687, aquí libro II (entero), pág. 93-283. Recuperado de http://www.aecid.es/ES/biblioteca/biblioteca-digital.

John Carter Brown Library - Indigenous Collection, https://archive.org/details/jcbindigenous.

Leal Feitosa Coelho, M. C. (2001). Catálogo da Coleção De Angelis. Anais da Biblioteca Nacional. Recuperado de http://objdigital.bn.br/acervo_digital/anais/anais.htm.

Maeder, E. (2001). El Martirologio romano, hallazgo del primer libro impreso en el Rio de la Plata. Idea Viva, Gaceta de Cultura, № 9, Marzo, pág. 17-18, 46.

(2008). Hallazgo en Bolivia del primer libro impreso en las misiones jesuíticas. Emprender en la Región, Revista de economia y negocios, Corrientes, Septiembre, Año 2, $\mathrm{N}^{\mathrm{o}} 35$, pág. 38. Recuperado de http://issuu.com/emprender_en_la_region/docs/emprender_n35.

Meier, J. (ed.) (2005-siguientes]. Jesuiten aus Zentraleuropa in Portugiesisch- und Spanisch-Amerika. Ein bio-bibliographisches Handbuch mit einem Überblick über das außereuropäische Wirken der Gesellschaft Jesu in der frühen Neuzeit. Münster: Aschendorff.

Meliá SJ, B. (1979). Alonso de Aragona, Breve introducción para aprender la lengua guarani, edición y notas por Amerindia 4., pág. 23-61, Paris. Recuperado de https://www.vjf.cnrs.fr/sedyl/amerindia/articles/pdf/A_04_02.pdf.

(1980). Alonso de Aragona, Primera gramática de la lengua guaraní, presentación, preparación material y notas por ... Posadas, Argentina: Ediciones Montoya.

(1992). La lengua guaraní del Paraguay, historia, sociedad y literatura, Madrid: Editorial MAPFRE. 
(1995). Etimología y semántica en un manuscrito inédito de Antonio Ruiz de Montoya (1651). La "découverte" des langues et des écritures d'Amérique (Amerindia 19/20.1995, congreso Paris 1993) Recuperado de https://www.vjf.cnrs.fr/sedyl/amerindia/articles/pdf/A_19-20_30.pdf

(2002). Un Guaraní reportero de guerra. En Meliá SJ, B. (ed.). Historia inacabada, futuro incierto; VIII Jornadas Internacionales sobre las Misiones Jesuíticas), Asunción, CEPAG, pág. 217-222.

Mitre, B. (1894). Lenguas americanas, estudio bibliográfico-lingüistico de las obras del P. Louis de Valdivia sobre el Araucano y el Allentiak, con un vocabulario razonado del Allentiak. La Plata 1894.

(1896). Orígenes de la imprenta argentina. La Biblioteca. Buenos Aires, 2.1896.52-57. Recuperado de http://www.biblioteca.org.ar/libros/656485.pdf.

(ed.) (1912). Lenguas americanas. Museo Mitre. Catálogo ilustrado de la Sección X de la Biblioteca. Buenos Aires: Coni.

Mitre, B. y Torres, L. M. (eds.) (1909-1911). Catálogo razonado de la Sección Lenguas Americanas Bartolomé Mitre. Con una introducción de Luis María Torres. Buenos Aires: Coni. 3 vol. Extracto Recuperado de http://www.museomitre.gov.ar/pdfs/Catalogo_lenguas_americanas.pdf.

Muñoz y Manzano, C. (conde de la Viñaza) (1892). Bibliografia española de lenguas indígenas de América. Por el conde de la Viñaza. Madrid: Est. Tipográfico "Sucesores de Rivadeneyra". Recuperado de http://bdh.bne.es/bnesearch/detalle/bdh0000065231.

N.N. (2015). Guarinihape tecocue - Lo que pasó en la guerra /1704-1705). Memoria anónima en guaraní del segundo desalojo de la Colonia del Santo Sacramento / Uruguay. Edición crítica del texto guaraní con traducción española, comentada por Harald Thun, Leonardo Cerno y Franz Obermeier. Kiel: Westensee-Verlag (Segunda edición corregida: Flor del Desierto 2016).

Neumann, E. S. (2005). Práticas letradas guaraní. Produção e usos da escrita indígena (séculos XVII e XVIII). Tesis doctoral. Rio de Janeiro: Universidade Federal do Río de Janeiro En:http://www.dominiopublico.gov.br/pesquisa/DetalheObraForm.do?select_act $\underline{\text { ion=\&co_obra }=107879 \text {. }}$

Obermeier, F. (2006). Der argentinische Erstdruck Nierembergs De la diferencia in Guaraní im Kontext der Bilderzyklen in Lateinamerika im 18. Jahrhundert. Online-Publikation UB Heidelberg, ART-Dok. Recuperado de http://archiv.ub.uni-heidelberg.de/artdok/154/1/obermeier.PDF.

(2011). Wie und mit welchen Mitteln erforschte man südamerikanische Indianersprachen. Wolfenbütteler Notizen zur Buchgeschichte 35:2.2011.167183. Recuperado de http://macau.uni-kiel.de/.

(2011). Wie und mit welchen Mitteln erforschte man südamerikanische Indianersprachen, in: Wolfenbütteler Notizen zur Buchgeschichte, 35, número 2, pág.167-183. Recuperado de http://macau.uni-kiel.de/.

(2015). Pedro de Angelis und seine Bedeutung für die Ethnolinguistik des La Plata-Raums, eine Rekonstruktion der bedeutendsten ethnolinguistischen 
Sammlung Argentiniens im 19. Jahrhundert. Wolfenbütteler Notizen. № 1, pág. 69-90. Recuperado de http://macau.uni-kiel.de/

(2017). Die Inventare der Jesuitenreduktionen bei der Vertreibung des Ordens aus dem La-Plata-Raum im 18. Jahrhundert und ihre buch- und bibliotheksgeschichtliche Bedeutung. Wolfenbütteler Notizen zur Buchgeschichte (en prensa).

Obermeier, F. y Cerno, L. (2013). Nuevos aportes de la lingüística para la investigación de documentos jesuíticos de los siglos XVII y XVIII. Folia Histórica del Nordeste. Resistencia (Chaco), 21, pág. 33-56.

Platzmann, J. (1876). Verzeichniss einer Auswahl amerikanischer Grammatiken, Wörterbücher, Katechismen. Leipzig: Köhler. Recuperado de https://archive.org.

Restivo, P. (1890) [1718]. Brevis Linguae Guarani Grammatica Hispanicae a Paulo Restivo secundum libros Antonii Ruiz de Montoya et Simonis Bandini... a 1718 composita et "Breve Noticia de la Lengua Guarani" inscripta. Christianus Fredericus Seybold (ed.), Stuttgart: Kohlhammer. Recuperado de https://archive.org.

(1892) [1724]. Linguae Guarani Grammatica Hispanice a Rev. P. Jesuita Paulo Restivo secundum libros Antonii Ruiz de Montoya, Simonis Bandini aliorumque adjecto Particularum lexico anno MDCCXXIV in Civitate S. Mariae Majoris. Christianus Fredericus Seybold (ed.). Stuttgart: Kohlhammer. Recuperado de https://archive.org.

(1893) [1722]. Lexicon Hispano-Guaranicum "Vocabulario de la lengua Guarani” inscriptum a Paulo Restivo secundum Vocabularium Antonii Ruiz de Montoya anno 1722. Christianus Fredericus Seybold (ed.). Stuttgard: Kohlhammer. Recuperado de https://archive.org.

Ruiz de Montoya SI, A. (1639). Conqvista espiritual hecha por los religiosos de la Compañia de Iesus, en las prouincias del Paraguay, Parana, Vruguay y Tape. Madrid: en la imprenta del Reyno Recuperdado de http://fondosdigitales.us.es/fondos/libros/1837/1/conquista-espiritual-hecha-porlos-religiosos-de-la-compania-de-iesus-en-las-provincias-del-paraguay-paranauruguay-y-tape/ Traducción de N.N. (Jaime Bonenti?) en Guarani, ms: Aba reta y caray eỹ baecue Tupã... Carátula: 1733 S. Nicolas, Colofone S. Ana, 04.05.1754, Rio: Biblioteca nacional. Ed.: Baptista Caetano de Almeida Nogueira como "Manuscripto Guarani sôbre a primitiva catechese dos Indios das Missões". Annaes da biblioteca nacional, Rio, Texto: tomo entero 6.1878/79, Vocabulario: tomo entero 7.1879/1880. Recuperado de http://objdigital.bn.br/acervo_digital/anais/anais.htm. El segundo ms. antes en Berlin, Staatsbibliothek, hoy Krakau, Biblioteka Jagiellońska. Recuperado de http://jbc.bj.uj.edu.pl/dlibra/

\section{Obras linguísticas de Ruiz de Montoya.}

1876 Ed. Julius Platzmann [1639/40]. Tomo 1: Arte de la lengua Guaraní, Tomo 2: Bocabulario de la lengua Guaraní, Tomo 3: Tesoro de la lengua Guaraní,. Tomo 4: Catecismo, 4. vol. Leipzig: Teubner, archive.org. 
1876, Ed. Francisco Adolfo Varnhagen [1639-1640]. Gramatica y diccionarios (arte, vocabulario y tesoro) de la lengua tupi ó guaraní. Nueva edición, mas correcta y esmerada que la primera. Viena: Frick; Paris: Maisonneuve, 2 tomos con títulos „Arte de la lengua guaraní ó mas bien tupi“, 2. parte: „Vocabulario y tesoro de la lengua guaraní o mas bien tupi, en dos partes“: I „Vocabulario español-guaraní (ó tupi)“; II „Tesoro guaraní (ó tupi)-español“",archive.org.

Para los originales en forma digital ver archive.org.

1993. Arte de la lengua guaraní (1640). Edición facsimilar con introducción y notas por Bartolomeu Melià. Transcripción actualizada del texto original por Antonio Caballos. Asunción: Centro de Estudios Paraguayos "Antonio Guasch".

1994. Arte y bocabvlario de la lengva gvaraní 1640. Tomo 1: Arte, y bocabvlario de la lengva gvarani: compvesto por el padre Antonio Ruiz, de la Compañia de Iesvs, facs. [Arte Madrid, 1640, Vocabulario 1639]; Tomo 2: Arte, primera parte. Estudio y transcripción, presentación, transliteración y notas de Silvio M. Liuzzi. Madrid: Ed. de Cultura Hispánica.

Sabor, J. E. (1995). Pedro de Angelis y los orígenes de la bibliografía argentina. Ensayo bio-bibliográfico. Buenos Aires: Ediciones del Solar.

Schmidel, U. (2008) [1554]. Viaje al Río de la Plata / Reise in die La Plata-Gegend. Franz Obermeier (ed.). Texto original del autógrafo de Stuttgart y traducción española de Edmundo Wernicke. Kiel: Westensee-Verlag.

Staden, H. 2007 [1557]. Warhaftige Historia. Zwei Reisen nach Brasilien (1548-1555) / História de duas viagens ao Brasil. Edicíón crítica port./alemán: Franz Obermeier. Kiel: Westensee-Verlag. [con facsimile de la edición princeps Warhaftige Historia und beschreibung eyner Landtschafft der Wilden Nacketen Grimmigen Menschenfresser Leuthen in der Newenwelt America gelegen, Marburg: Kolbe colofone "uff Fastnacht" 1557].

Storni SJ, Hugo (1980), Catalogo de los Jesuitas de la provincia del Paraguay (Cuenca del Plata) 1585-1768, Roma: Institutum Historicum.

Telesca, Ignacio (2006). Documentos jesuíticos del siglo XVIII en el Archivo Nacional de Asunción. Asunción: Centro de Estudios Paraguayos Antonio Guasch, CEPAG 2006

Valdivias, Luis (1607). Doctrina Christiana y cathecismo en la lengua Allentiac, que corre en la ciudad de S. Iuan dela Frontera, con vn Confessonario, Arte, y Bocabulario breues. Lima: por Francisco del Canto.

Valle Cabral, A. do. (1881). Bibliographia das obras tanto impressas como manuscriptas, relativas á lingua tupi ou guarani tambem chamada lingua geral do Brazil. Annaes da Biblioteca Nacional. VIII, 1880/1881, Rio 1881.143-214. Recuperado de https://archive.org/

Weigel, Oswald.(ed.). 1903. Verzeichnis der werthvollen, an Seltenheiten reichen Bibliothek des verstorbenen Amerikanisten Julius Platzmann, Oswald Weigel's Bücher-Auction. Leipzig: Oswald Weigel. 
Manuscritos [selección]

Angelis, P. de. Ms. Archivo General de la Nación en Buenos Aires, Archivo y Colección Andrés Lamas (1549-1894); Legajos 22 y 23, en ese ultimo "Estudios y vocabulario de la lengua Guaraní“.

N.N. "Diário hecho por un indio de lo que sucedió en el segundo desalojamiento de los portugueses [de la Colonia de Sacramento] en 1o de septiembre de 1704 [hasta el 18 de marzo de 1705, Título posterior, probablemente por Pedro de Angelis] copia en la colección privada de Alejandro Larguía, Posadas, para la edición ver: N.N. Guarinihape tecocue - Lo que pasó en la guerra.

N.N. "Abschrift eines im Privatbesitz des Herrn von Gülich befindlichen handschriftlichen Guaraní-Fragmentes: im Auftrage von Julius Platzmann für Herrn Dr. Karl Henning, angefertigt durch Emanuel Forchhammer, Leipzig, 1878”. Ms. Colección Brinton, Penn Library, University of Pennsylvania, Museum of Archaeology and Anthropology, Philadelphia, hoy en la sección de libros raros de esa biblioteca. Recuperado de http://dla.library.upenn.edu/dla/medren/detail.html?id=MEDREN_4226236. 